\title{
Volatile organic compounds stimulate plant growing and seed germination of Lactuca sativa
}

\author{
Paola Fincheira ${ }^{1,}$ Maribel Parada ${ }^{2}$ and Andrés Quiroz ${ }^{1 *}$
}

${ }^{1}$ Centro de Excelencia en Investigación Biotecnológica Aplicada al Medio Ambiente, Facultad de Ingeniería y Ciencias, Universidad de La Frontera. Av. Francisco Salazar 01145, Casilla 54-D, Temuco, Chile. ${ }^{2}$ Centro Biotecnológico de Estudios Microbianos, Facultad de Ciencias Agronómicas y Forestales, Universidad de La Frontera. Av. Francisco Salazar 01145, Casilla 54-D, Temuco, Chile. "Corresponding autor: andres.quiroz@ ufrontera.cl

\begin{abstract}
Volatile organic compounds (VOCs) emitted by Bacillus species have been reported as growth inducers in Arabidopsis thaliana, but their effects on horticultural species have been scarcely studied. In this study, Lactuca sativa emerges as a model vegetable to evaluate VOCs release by Bacillus sp. BCT9. The results indicated that root length, dry weight, number of lateral root and shoot length increased after VOCs exposition. The initial application of $30 \mu \mathrm{L}$ of BCT9 in Nutrient Agar (N-A) was the best dose to elicit growth; whereas $60 \mu \mathrm{L}$ of BCT9 inoculated in Methyl Red \& Voges Proskauer Agar (MRVP-A) and Murashige \& Skoog Agar (MS-A) had a greater effect. It noteworthy that root development was higher when BCT9 was grown in MRVP-A than in the others culture medium. The identified VOCs released by BCT9 in MRVP-A were 3-hydroxy-2-butanone, 2,3-butanediol, 2-nonanone, 2-undecanone, 2-tridecanone and 2-pentadecanone. Furthermore, the toxicity bioassays indicated that most VOCs did not have high toxic effects and some VOCs stimulated the growth at seed germination stage. In conclusion, this study suggests that VOCs can strongly modulate the L. sativa growth during germination and seedlings stages, so new explorations should be carried out in other vegetables to determine their effects.
\end{abstract}

Keywords: Culture conditions, growth modulation, volatile organic compounds (VOCs), phytotoxicity 


\section{Introduction}

Volatile organic compounds (VOCs) are molecules with low molecular weight $(300 \mathrm{~g} / \mathrm{mol})$ and high vapour pressure $\left(0.01 \mathrm{kPa}\right.$ at $\left.20^{\circ} \mathrm{C}\right)$ that include diverse chemical compounds (i.e. ketones). In the last decade, VOCs emitted by species belonging to Bacillus genus have been described for their ability to induce growth in Arabidopsis thaliana, which is usually used as model plant (Ryu et al., 2003; Kanchiswamy et al., 2015). The VOCs have the ability to elicit plant growth in absence of physical contact through the induction of physiological changes depending on doses and culture medium for bacterial growth (Zhang et al., 2007; Blom et al., 2011). Therefore, we propose that VOCs emitted by Bacillus species can be a new strategy to induce growth on horticultural species for reducing the application of agrochemical products. Based on the above mentioned, Lactuca sativa emerges as a model vegetable to test volatiles as growth inducer due to easy management, fast germination and sensitivity to compounds exposition (Charles et al., 2011). The objectives of the present study were: (1) to evaluate culture conditions of Bacillus sp. BCT9 for producing volatiles with growth-inducing activity and (2) to determine the effects of identified volatile organic compounds on L. sativa germination.

\section{Materials and Methods}

\subsection{Bacterial isolates and plant growth conditions}

The Bacillus sp. BCT9 (Genbank access number: KX395632) was streaked on Plate Count Agar. Commercial seeds of L. sativa (Green lettuce cv Reina de mayo asepo, semillas Fito, S.A) were surfacesterilized during $8 \mathrm{~min}$ with $3 \%$ sodium hypochlorite and washed with sterile distilled water. Later, seeds were placed on Murashige and Skoog basal medium with vitamins 0.5X (PhytoTechnology Laboratories, LLC ${ }^{\mathrm{TM}}$ ) containing $0.8 \%$ agar and $1.5 \%$ sucrose (MS-A). Petri dishes were placed under 16/8-h lightdark cycle at $20-25{ }^{\circ} \mathrm{C}$. Germinated seedlings were transferred to two-compartment Petri dishes after 2 days for experimental uses (Ryu et al., 2003).

\subsection{Evaluation of Bacillus sp. BCT9 dose on L. sa- tiva growth}

According to the methodology reported by Fincheira et al. (2016), bioassays were performed in two-compartment Petri dishes $(90 \times 15 \mathrm{~mm})$ with two 2-day-old L. sativa seedlings placed into one of the compartments containing MS-A and the second compartment containing Nutrient agar (N-A), Methyl Red \& Voges Proskauer agar (MRVP-A) or MS-A. The second compartment was inoculated with 15,30 or $60 \mu \mathrm{L}$ of Bacillus sp. BCT9 $(2 \mathrm{x}$

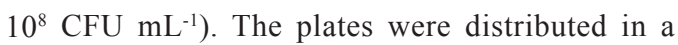
randomized design and non-inoculated plates were used as control (Blom et al., 2011; VelázquezBecerra et al., 2011). The evaluation of L. sativa growth was measured on day- 10 .

\subsection{GC-MS analysis of volatile organic compounds released by Bacillus sp. BCT9}

Bacterial isolate $(25 \mathrm{~mL})$ was grown in $250 \mathrm{~mL}$ of MRVP for $19 \mathrm{~h}$ at $34{ }^{\circ} \mathrm{C}$ to collect volatiles using Solid Phase Micro Extraction (SPME) fiber polydimethylsiloxane/divinylbenzene (PDMS/ DVB), previously conditioned with helium for 10 min at $250{ }^{\circ} \mathrm{C}$. Volatiles were desorbed at $250{ }^{\circ} \mathrm{C}$ for $2 \mathrm{~min}$ in an injector of gas chromatograph coupled with mass spectrometer (Thermo Electron Corporation). The chromatographic separation was per- 
formed by DB-1 column using helium flow $(1.0 \mathrm{~mL}$ $\mathrm{min}^{-1}$ ). Mass spectra were acquired from 35 to 500 a.m.u applying an electronic imput of $70 \mathrm{eV}$.The VOCs were identified by comparing Kovats indices (KIs) with the corresponding commercial standards by injecting an alkane series (C9-C26) (Tampe et al., 2016).

\subsection{Germination toxicity assays}

The disinfected seeds $(n=15)$ were placed on the surface of MS-A in one of the side of two-compartment Petri dishes and exposed to different doses $(1,000$ $\mu \mathrm{g}, 1 \mu \mathrm{g}$ and $0.01 \mu \mathrm{g}$ ) of identified volatile organic compounds placed in the other compartment during germination stage. Three plates were used to test each combination (compound $\mathrm{x}$ concentration). Root elongation (RE) (RE=Elongation sample $^{- \text {Elonga- }}$ tion $_{\text {control }} /$ Elongation $_{\text {control }}$ ) and seed germination (SG) ( $\mathrm{SG}=$ Germination $_{\text {sample }^{-}}$- Germination control $_{\text {Germina- }}$ tion $_{\text {control }}$ ) were evaluated as toxicity parameters. The indices are designed with values in a range from -1 (maximum phytotoxicity) to $>0$. The toxicity effect was evaluated after $120 \mathrm{~h}$ according to the following scale: (1) 0 to $-0.25=$ low toxicity, (2) -0.25 to $-0.5=$ moderate toxicity, (3) -0.5 to $-0.75=$ high toxicity and (4) -0.75 to $-1=$ very high toxicity. A value more than zero indicate growth stimulation (BagurGonzález et al., 2010).

\subsection{Statistical analysis}

The growth parameters were analyzed by Statistix v10. The diferent results from the VOCs treatments on L. sativa growth parameters were analyzed using analysis of variance (ANOVA) and LSD test (P $\leq 0.05)$.

\section{Results}

3.1. The effects of different culture conditions on $B a$ cillus sp. BCT9 to release VOCs as growth modulator on L. sativa

Figure 1 shows that seedlings exhibits 24, 54, 51 and $190 \%$ increase in root length, dry weight, shoot length and number of lateral roots, respectively, when BCT9 $(30 \mu \mathrm{L})$ was grown in $\mathrm{N}-\mathrm{A}$.

Nevertheless, the exposition of seedlings to BCT9 VOCs $(60 \mu \mathrm{L})$ grown in MRVP-A shows increased root length (80\%) (Figure 1a). L. sativa seedlings showed a 98 and $58 \%$ increase in dry weight and shoot length, respectively (Figure 1b,d). In addition, dry weight and number of lateral roots increased 127 and $95 \%$, respectively when BCT9 $(60 \mu \mathrm{L})$ was cultivated in MS-A (Figure 1b). Furthermore, the number of lateral roots increased more than 6-fold compared with the control (Figure 1c). For the next experiments the MRVP medium was chosen to identify VOCs emitted by BCT9 due to its highest capacity to elicit an increase of root length.

\subsection{The effect of VOCs release by BCT9 on seeds ger-} mination of L. sativa.

The identified compounds released from BCT9 grown in Methyl Red \& Voges Proskauer were 3-hydroxy2-butanone, 2,3-butanediol, 2-nonanone, 2-undecanone, 2-tridecanone and 2-pentadecanone. The VOCs showed a low toxicity on $L$. sativa, according to indices from - 0.27 to 0 , only 2 -nonanone at $1000 \mu \mathrm{g}$ presented toxicity. Ketone compounds increased both RE and SG in at least one applied concentration. Finally, it noteworthy that 2-nonanone $(0.1 \mu \mathrm{g}), 2$-undecanone $(1 \mu \mathrm{g})$ and 2-tridecanone $(0.01$ and $1 \mu \mathrm{g})$ stimulated the growth on L. sativa at germination stage (Table 1). 

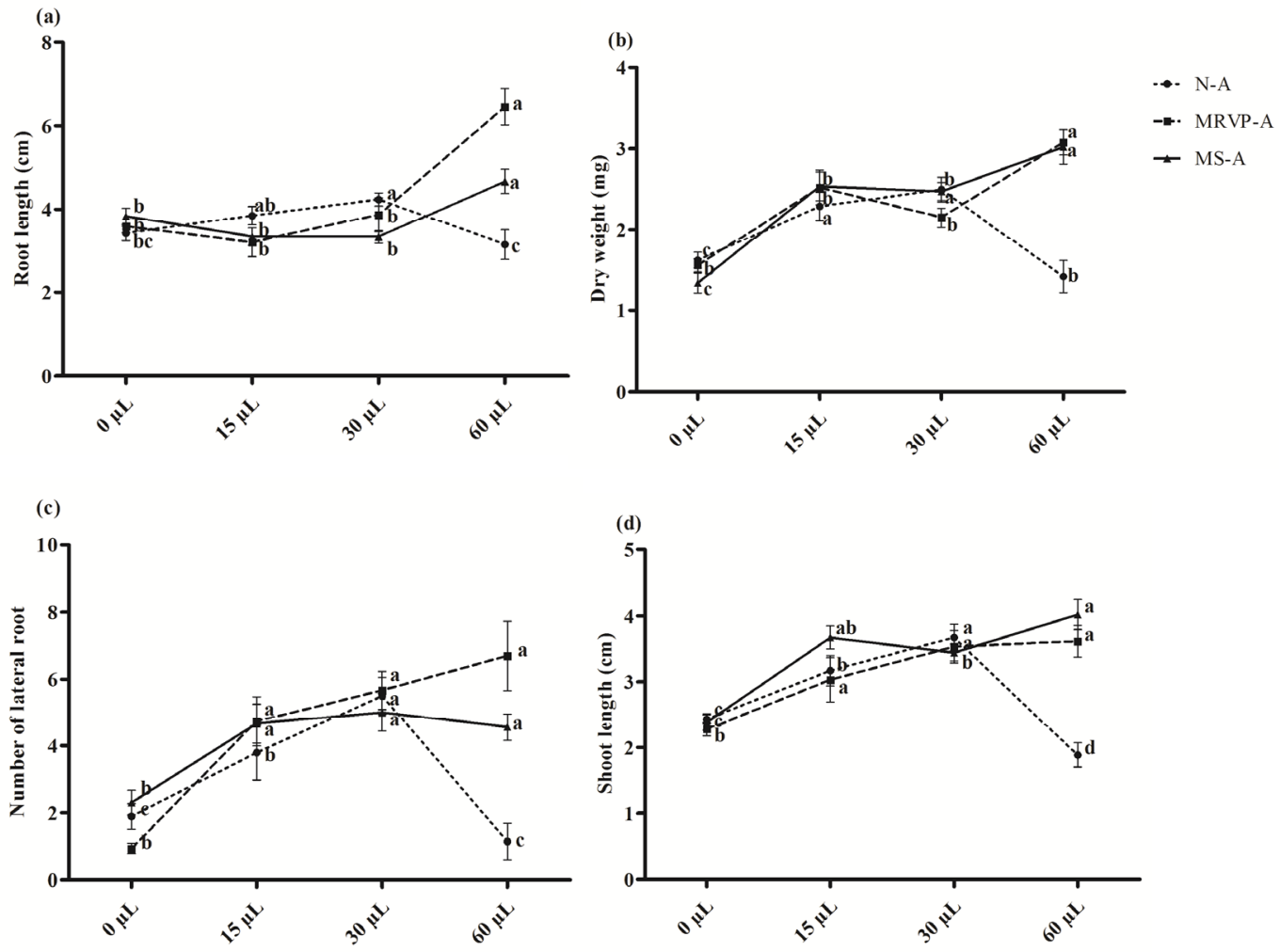

Figure 1. Effects of different doses of Bacillus sp. BCT9 on the emission of volatile organic compounds on growth modulation of Lactuca sativa. The growth parameters evaluated were (a) root length, (b) dry weight, (c) number of lateral root and (d) shoot length. N-A= Nutrient agar; MRVP-A= Methyl Red \& Voges Proskauer agar; MS-A = Murashige \& Skoog agar. Bars represent the standard error. Letters indicate means that differ significantly according to ANOVA (LSD test) for each culture medium $(\mathrm{P}<0.05)(\mathrm{N}=15-20)$. 
Table 1. Identified volatile organic compounds released from Bacillus sp. BCT9 cultivated in MRVP medium and their effects on germination $(\mathrm{RT}=$ retention time, $\mathrm{RL}=$ root length, $\mathrm{Germ}=$ germinated seeds, $\mathrm{RE}=$ root elongation, $\mathrm{SG}=$ seed germination). Letters indicate means that differ significantly according to ANOVA (LSD test) (N=3).

\begin{tabular}{lclcccc}
\hline Compound & RT (min) & Doses $(\boldsymbol{\mu g})$ & RE $(\mathbf{c m})$ & Germ $(\%)$ & RE & SG \\
\hline 3-Hydroxy-2-butanone & 3.9 & 1000 & $0.46 \pm 0.17^{\text {ef }}$ & 97 & -0.16 & -0.01 \\
& & 1 & $0.49 \pm 0.16^{\text {ef }}$ & 90 & -0.10 & -0.08 \\
& & 0.01 & $0.48 \pm 0.16^{\text {ef }}$ & 97 & -0.12 & -0.01 \\
\hline 2,3-Butanediol & 5.1 & 1000 & $0.44 \pm 0.14^{\mathrm{f}}$ & 95 & -0.2 & -0.03 \\
& & 1 & $0.46 \pm 0.15^{\text {ef }}$ & 97 & -0.16 & -0.01 \\
& & 0.01 & $0.50 \pm 0.16^{\text {def }}$ & 98 & -0.09 & 0 \\
\hline 2-Nonanone & 13.0 & 1000 & $0.40 \pm 0.15^{\text {f }}$ & 23 & -0.27 & -0.76 \\
& & 1 & $0.59 \pm 0.23^{\text {bc }}$ & 95 & 0.07 & -0.03 \\
& & 0.01 & $0.57 \pm 0.19^{\text {bcd }}$ & 98 & 0.03 & 0 \\
\hline 2-Undecanone & 18.7 & 1000 & $0.61 \pm 0.19^{\mathrm{b}}$ & 87 & 0.10 & -0.11 \\
& & 1 & $0.69 \pm 0.24^{\text {a }}$ & 98 & 0.25 & 0 \\
\hline 2-Tridecanone & & 0.01 & $0.53 \pm 0.18^{\text {cde }}$ & 80 & -0.03 & -0.18 \\
\hline 2-Pentadecanone & 1000 & $0.63 \pm 0.23^{\text {ab }}$ & 90 & 0.14 & -0.08 \\
& 23.9 & 1 & $0.60 \pm 0.17^{\text {bc }}$ & 98 & 0.09 & 0 \\
\hline
\end{tabular}

\section{Discussion}

Bacterial species have been intensively studied for their ability to increase plant growth by emission of non-volatile compounds, but recent studies have discussed strongly the relevant ecological role of volatiles by their ability to induce growth without physical contact (Casarrubia et al., 2016; Tyc et al., 2016). In the last decade, Ryu et al. (2003) reported that VOCs emitted by Bacillus subtilis G03 can elicit growth in A. thaliana through the activation of physiological pathways. Nevertheless, culture conditions for bacterial growth have an essential role for the emission of volatiles such as inoculums quantity and culture medium composition (Blom et al., 2011; Velázquez-Becerra et al., 2011). In this study, different culture conditions of Bacillus sp. BCT9 were evaluated for producing VOCs as growth modulators on $L$. sativa. The VOCs produced by BCT9 elicited $L$. sativa growth depending strongly on inoculated doses, in agree with the reports by Velázquez-Becerra et al. (2011) and Blom et al. (2011), who indicated that low doses of inoculums elicit growth, and high doses can induce phytotoxicity in seedlings. Recently, the $B a$ cillus genus was studied by Asari et al. (2016), who showed that VOCs released by different strains (Coded as UCMB5033, UCMB5036, UCMB5113 and FZB42) induced a significant increase on dry weight of $A$. thaliana (phyllosphere) when the strains grow in Luria Broth Agar (LB-A), minimal medium (M9) or Trypticase Soy Agar, indicating that doses of $B a$ cillus amyloliquefaciens UCMB5113 from 20 to 100 $\mu \mathrm{L}$ inoculated in MS-A increased dry weight (phyllosphere). Besides, Blom et al. (2011) reported that Burkholderia pyrrocinia Bcc171 increased dry weight on A. thaliana when grown in LB-A and MRVP-A, reaching the best yield with $10 \mu \mathrm{L}$ of applied inoculums. Furthermore, Medicago sativa - Arthrobacter agilis UMCV2 interaction was studied by VelázquezBecerra et al. (2011), who reported a dose-dependence 
response of $M$. sativa exposed to VOCs release by $A$. agilis UMCV2, reaching the best increase on root length, root density, stem length and fresh weight with $50 \mu \mathrm{L}$ of inoculum grown in N-A compared with doses from 100 to $500 \mu \mathrm{L}$. The studies described above suggest that low inoculums amount (range from 10 to 50 $\mu \mathrm{L})$ have a great effect to induce growth. The mentioned studies demonstrated the strong effect of dose dependence of bacterial inoculum (independent of the involved bacterial genus). In this study, it was shown that VOCs released by BCT9 have a relevant role to elicit the root growth, coinciding with the reported by Gutiérrez-Luna et al. (2010) and Meldau et al. (2013), who showed the importance of VOCs released by $\mathrm{Ba}$ cillus to elicit primary root length, lateral root number and lateral root length. The root development is essential to absorb nutrients and water from substrate; so many studies have focused on these parameters (Salazar-henao et al., 2016). Until now, mycorrhiza associations have been studied intensively for decades by its role to tolerate biotic and abiotic stress, plant productivity, nutrients acquisition and plant productivity (Van der Heijden et al., 2015). Specifically, a recent study performed by Durán et al. (2016) showed that the symbiotic interaction that involve arbuscular mycorrhizal fungi (Rhizophagus intraradices) applied together with Bacillus sp., Klebsiella sp. or Acinetobacter sp. in L. sativa can increase photosynthetic pigments and antioxidant enzyme levels under drought stress. In this study, the evaluation of physiological effects under stress was not performed, but action mechanisms associated to VOCs emitted by Bacillus have shown their ability to modulate essential nutrients concentration, hormonal balance, metabolisms and sugar concentrations after $48 \mathrm{~h}$ of exposition (Zhang et al., 2007). Therefore, VOCs can modulate diverse cellular target after short time of exposure with similar effects compared with symbiotic interaction, but this new mechanisms represents an ecological advantage because it can activate its target at long distance. The BCT9 grown in MRVP, showed a high ability to induce $L$. sativa growth, so this medium was selected to identify VOCs. The VOCs released are derived from two metabolic pathways: piruvate fermentation (3-hydroxy-2-butanone and 2,3-butanediol) and fatty acid cycle (2-nonanone, 2-undecanone, 2-tridecanone and 2-pentadecanone). Respect to germination assays, the results indicated that both indices showed a sensitivity to evaluate VOCs phytotoxicity and compounds showed a low toxicity on $L$. sativa during germination stage. Remarkably, the results indicated that 2-nonanone, 2-undecanone and 2-tridecanone had the ability to stimulate seed germination, suggesting their important influence in this stage, but more studies should be performed. This research shows the importance of culture conditions to prospect non-toxic VOCs as growth inducers in horticultural species to study VOCs as a new strategy to reduce agrochemical application.

\section{Conclusions}

VOCs released by Bacillus sp. BCT9 act as growth inducer agents at shoot and root level on L. sativa, obtaining the best yield with exposition to VOCs released in a range from 30 to $60 \mu \mathrm{L}$ of inoculums depending on culture conditions. In addition, the results suggest that VOCs have low toxicity effect on seeds and ketone compounds have stimulating effect on germination stage.

\section{Acknowledgements}

The authors thank CONICYT scholarship (21120145) and project Fondecyt (1141245) for their support in this work. Likewise, we also thank Dr. Maribel Parada for her kind help facilitating bacterial strains. Supported (partially) by Dirección de Investigación, Universidad de La Frontera. 


\section{References}

Asari, S., Matzén, S., Agerlin Petersen, M., Bejai, S., Meijer, J. 2016. Multiple effects of Bacillus amyloliquefaciens volatile compounds: plant growth promotion and growth inhibition of phytopathogens. Fems Microbiol. Ecol. 92: 6.

Bagur-González, M., Estepa-Molina, C., MartínPeinado, F., Morales-Ruano, S. 2010. Toxicity assessment using Lactuca sativa $\mathrm{L}$. bioassays of the metal(loid)s $\mathrm{As}, \mathrm{Cu}, \mathrm{Mn}, \mathrm{Pb}$ and $\mathrm{Zn}$ in solublein-water satured soil extracts from an abandoned mining site. J. Soils Sediments. 11, 281-289.

Blom, D., Fabbri, C., Connor, E., Schiestl, F., Klauser, D., Boller, T. ., Eberl, L., Weisskopf L. 2011. Production of plant growth modulating volatiles is widespread among rhizosphere bacteria and strongly depends on culture conditions. Environ. Microbiol. 13, 3047- 3058.

Casarrubia, S., Sapienza, S., Fritz, H., Daghino, S., Rosenkranz, M., Schnitzler, J., Martin, F., Perotto, S., Martino, E. 2016. Ecologically different fungi affect Arabidopsis development: Contribution of soluble and volatile compounds. Plos One. 11, e0168236.

Charles, J., Sancey, B., Morin-Crini, N., Badot, P., Degiorgi, F., Trunfio, G, Crini, G. 2011. Evaluation of the phytotoxicity of polycontaminated industrial effluents using the lettuce plant (Lactuca sativa) as a bioindicator. Ecotoxicol. Environ. Saf. 74, 2057-2064.

Durán, P., Acuña, J.J., Armada, E., López-Castillo, O.M., Cornejo, P., Mora, M.L., R, Azcón. 2016. Inoculation with selenobacteria and arbuscular mycorrhizal fungi to enhance selenium content in lettuce plants and improve tolerance against drought stress. J. Soil Sci. Plant Nutr. 16, $201-$ 225.
Fincheira, P., Venthur, H., Mutis, A., Parada, M., Quiroz, A. 2016. Growth promotion of Lactuca sativa in response to volatile organic compounds emitted from diverse bacterial species. Microbiol. Res. 193, 39-47.

Gutiérrez-Luna, F., López, J., Altamirano, J., Valencia, E., Reyes de la Cruz, H., Macías, L. 2010. Plant growth- promoting rhizobacteria modulate root- system architecture in Arabidopsis thaliana through volatile organic compound emission. Symbiosis. 51, 75- 83.

Kanchiswamy, C., Malnoy, M., Maffei, M. 2015. Bioprospecting bacterial and fungal volatiles for sustainable agriculture. Trends Plant Sci. 20, 206211.

Meldau, D., Meldau, S., Hoang, L., Underberg, S., Wünsche, H., Baldwin, I. 2013. Dimethyl disulfide produced by the naturally associated bacterium Bacillus sp B55 promotes Nicotiana attenuata growth by enhancing sulfur nutrition. Plant Cell. 25, 2731-2747.

Ryu, C., Farag, M., Hu, C., Reddy, M., Wei, H., Paré, P., Kloepper, J. 2003. Bacterial volatiles promote growth in Arabidopsis. Proc. Natl. Acad. Sci. U.S.A. 100, 4927- 4932.

Salazar-Henao, J., Vélez- Bermúdez, I., Schmidt, W. 2016. The regulation and plasticity of root hair patterning and morphogenesis. Development. 143, 1848-1858.

Tampe, J., Parra, L., Huaiquil, K., Quiroz, A. 2016. Potential repellent activity of the essential oil of Ruta chalepensis (linnaeus) from Chile against Aegorhinus superciliosus (guérin) (Coleoptera: Curculionidae). J. Soil Sci. Plant Nutr. 16, 48-59.

Tyc, O., Song, C., Dickschat, J.S., Vos, M., Garbeva, P. 2016. The ecological role of volatile and soluble secondary metabolites produced by soil bacteria. Trends Microbiol. 25, 280-292. 
Van der Heijden, M.G., Martin, F.M., Selosse, M.A., Sanders, I.R. 2015. Mycorrhizal ecology and evolution: the past, the present, and the future. New Phytol. 205, 1406-1423.

Velázquez-Becerra, C., Macías, L., Bucio, J., Altamirano, J., Flores, I., Valencia, E. 2011. A volatile organic compound analysis from Arthrobacter agilis identifies dimethylhexadecylamine, an aminocontaining lipid modulating bacterial growth and Medicago sativa morphogenesis in vitro. Plant Soil. 339, 329- 340.
Zhang, H., Kim, S., Krishnamachari, V., Payton, P., Sun, Y., Grimson, M., Farag, M., Ryu, C., Allen, R., Melo, I., Paré, P. 2007. Rhizobacterial volatile emissions regulate auxin homeostasis and cell expansion in Arabidopsis. Planta. 226, 839- 851. 
